\title{
ZWEI DIMENSIONEN DER INTERNATIONALISIERUNG: EINE EMPIRISCHE ANALYSE DEUTSCHER GROSSUNTERNEHMEN
}

\author{
Anke Hassel, Martin Höpner, Antje Kurdelbusch, Britta Rehder \\ und Rainer Zugehör
}

\author{
Article by an MPIfG researcher \\ Anke Hassel, Martin Höpner, Antje Kurdelbusch, Britta Rehder, Rainer Zugehör: Zwei Dimensionen der Internationalisierung: \\ Eine empirische Analyse deutscher Großunternehmen. \\ In: Kölner Zeitschrift für Soziologie und Sozialpsychologie 52(3), 500-519 (2000). VS Verlag für Sozialwissenschaften \\ The original publication is available at the publisher's web site: http://dx.doi.org/10.1007/s11577-000-0070-2
}

Zusammenfassung: Um den Einfluß wirtschaftlicher Internationalisierung auf nationale Institutionengefüge zu überprüfen, werden geeignete Messverfahren zur Messung von Internationalisierung benötigt. Der Beitrag stellt ein Verfahren zur Messung der Internationalisierung von Unternehmen vor. Dabei wird davon ausgegangen, dass die Internationalisierung von Unternehmen mehrere unterscheidbare Dimensionen hat. Die realwirtschaftliche Dimension beschreibt die güter- und produktionswirtschaftliche grenzüberschreitende Expansion der Unternehmen, während die kapitalmarktbezogene Dimension die Orientierung der Unternehmen an internationalen Kapitalmärkten abbildet. Anhand einer Untersuchung über den Internationalisierungsgrad der 100 größten deutschen Unternehmen werden beide Internationalisierungsdimensionen empirisch überprüft. Die Faktorenanalyse unterstützt die Annahme, dass sich beide Dimensionen empirisch deutlich voneinander unterscheiden lassen. Anhand der vorgestellten Messmethoden lassen sich die Unternehmen eindeutig in stark und schwach internationalisierte Unternehmen einteilen.

\section{Einleitung}

Ursachen, Prozesse und Auswirkungen der Internationalisierung werden zunehmend zu einem zentralen Fokus wissenschaftlicher und politischer Debatten. Insbesondere die wirtschaftliche Internationalisierung hat weit reichende Konsequenzen für die Handlungsfähigkeit nationaler Akteure sowie für den Wandel national begrenzter Institutionensysteme (Dörre 1997; Scharpf 1997; Streeck 1998). Wirtschaftliche Internationalisierung bezieht sich auf die Ausweitung von Märkten für Produkte und Vorprodukte, Arbeit (einschließlich im Ausland erbrachter produktiver Vorleistungen) und Kapital (Kredite ebenso wie Unternehmensanteile) über Grenzen hinweg, also über die Reichweite nationaler Institutionen hinaus. Es wird vermutet, dass die internationale Durchdringung von Märkten für Produkte und Kapital den Druck auf nationale Institutionensysteme steigert, sich entweder den neuen Bedingungen der Kapitalverwertung anzupassen oder empfindliche Einbußen an Investorentätigkeit in ihren Ländern hinnehmen zu müssen.

Wie sich der aus der wirtschaftlichen Internationalisierung entstehende Wettbewerb zwischen nationalen institutionellen Systemen auf deren Wandel sowie auf die Handlungsfähigkeit nationaler Akteure auswirkt, ist weitgehend unklar. Oftmals wird argumentiert, die zunehmende internationale Mobilität von Kapital und Produktion stärke die Stellung von Arbeitgebern, die ihren Machtzuwachs für eine Flexibilisierung von vormals rigiden und zentralisierten Regulierungen auf dem Arbeitsmarkt nutzen kön- 
nen (Kapstein 1996). Andererseits lassen sich Anzeichen dafür finden, dass die aus der Internationalisierung erwachsene Empfindlichkeit gegenüber Produktionsausfällen den Arbeitgebern die Durchsetzung ihrer Forderungen gegenüber den Gewerkschaften erschwert (Thelen 1999).

Gleichzeitig gibt es deutliche Anzeichen dafür, dass sich die Form der wirtschaftlichen Internationalisierung wandelt. Während sich in der Vergangenheit grenzüberschreitende wirtschaftliche Aktivitäten im Wesentlichen auf Direktinvestitionen multinationaler Unternehmen zur Produktion von Gütern sowie auf den grenzüberschreitenden Handel mit Gütern beschränkte, gibt es heute darüber hinaus grenzüberschreitenden Handel mit Eigentumsrechten an Unternehmen und einen weltweit weitgehend liberalisierten Kapitalmarkt. Beides hat Auswirkungen für das traditionelle Verständnis wirtschaftlicher Internationalisierung. So besteht der überwiegende Anteil ausländischer Direktinvestitionen bereits in den neunziger Jahren aus der Übernahme bestehender Unternehmen in dem Empfängerland (Wortmann 2000). Zum Beispiel ist die Übernahme von Mannesmann durch Vodafone voraussichtlich im Umfang die größte Direktinvestition aus Großbritannien nach Deutschland im Jahr 2000, ohne dass sich an der wirtschaftlichen Aktivität des Mannesmannkonzerns notwendigerweise etwas ändern müsste. Zudem nutzen Unternehmen zunehmend internationale Kapitalmärkte zur Finanzierung ihres Unternehmens. Die Orientierung deutscher Großunternehmen an internationalen Kapitalmärkten ändert die Eigentümerstrukturen dieser Unternehmen durch die Öffnung an internationale Investoren und die Umstellung der Rechnungslegung auf internationale Standards. Beide Prozesse - die Entwicklung eines internationalen Marktes für Unternehmensübernahmen und die Öffnung nationaler Unternehmen für internationale Investoren - werden in der bestehenden Internationalisierungsdiskussion kaum berücksichtigt. Insbesondere die Frage, ob es sich dabei um eine weitere Stufe bereits internationalisierter Unternehmen oder um eine eigenständige Dimension wirtschaftlicher Internationalisierung handelt, ist bislang noch kaum thematisiert worden.

Der vorliegende Artikel möchte einen konzeptuellen wie auch empirischen Beitrag zur Operationalisierung und Messung der Internationalisierung von Unternehmen leisten. Im ersten Teil wird der Stand der methodischen Debatte in der empirischen Internationalisierungsforschung reflektiert. Anschließend wird ein Konzept zur Messung der Internationalisierung deutscher Unternehmen vorgeschlagen, und erste Ergebnisse werden präsentiert. Wir argumentieren, dass sich mit einem realwirtschaftlichen und einem kapitalmarktbezogenen Index zwei Dimensionen der Internationalisierung unterscheiden lassen, die theoretisch voneinander unterschieden werden müssen und die empirisch nur schwach miteinander korrelieren. 


\section{Die Messung von Internationalisierung}

Im folgenden Abschnitt werden die Erfolge und Probleme bisheriger Messversuche diskutiert. Die zu diesem Thema bestehenden Arbeiten unterscheiden sich hinsichtlich ihres Aggregationsniveaus, ihrer Indikatorenauswahl sowie hinsichtlich ihres Verfahrens der Indikatorenbündelung. ${ }^{1}$

\section{Aggregationsebene}

Ein Teil der Literatur nimmt die Internationalisierung von Volkswirtschaften anhand makroökonomischer Kennziffern über Außenhandelsquoten und Direktinvestitionen zum Ausgangspunkt der Analyse (Hirst und Thompson 1996; Keohane und Milner 1996; Scharpf 1997; Streeck 1998). Dieses Vorgehen setzt die Annahme voraus, alle am Wirtschaftsprozess beteiligten Unternehmen seien in gleicher Weise von der wirtschaftlichen Internationalisierung betroffen. Es gibt jedoch Hinweise darauf, dass dies nur für die passive wirtschaftliche Internationalisierung (wie z.B. die Importquote) gilt und auch das nur sektorenspezifisch. Die aktive Internationalisierung wird hingegen unternehmensspezifisch gestaltet (Bartlett 1986). Vor diesem Hintergrund scheint es wichtig, Internationalisierung auch anhand unternehmensspezifischer Indikatoren zu messen. Zwei weitere Gründe sprechen dafür:

Erstens sind Unternehmen die wichtigsten Akteure der wirtschaftlichen Internationalisierung. Sie exportieren einen erheblichen Teil ihrer Produktion, verfügen in wachsendem Maße über ausländische Produktionsstätten und Belegschaften und müssen ihr Kapital auf internationalen Märkten beschaffen. Daher ist wirtschaftliche Internationalisierung mit einer wachsenden Intensität des Wettbewerbs innerhalb und zwischen Unternehmen verbunden: um Absatzmärkte, um Arbeitsplätze, um Investitionskapital.

Zweitens sind Unternehmen, und hier vor allem die großen Unternehmen, wesentliche Gestalter und Empfänger von Regulierung innerhalb des Systems der industriellen Beziehungen. Großunternehmen haben hinsichtlich der Gestaltung immer eine führende Rolle gespielt, vor allem auf Grund ihrer starken Stellung in den Verbänden der Arbeitgeber. Die Bedeutung großer Unternehmen als strategische Akteure wird im Bereich der industriellen Beziehungen seit den achtziger Jahren betont (Kochan et al. 1986). Ob die großen deutschen Unternehmen weiterhin bereit und in der Lage sind, ihre Interessen kollektiv im Rahmen von verbandlicher Interessenvermittlung zu verfolgen, oder ob sie auf eine Internalisierung und damit eine strategische Individualisierung ihrer Arbeitspolitik setzen werden, ist eine der wichtigsten Fragen in der Debatte um die Zukunft des Modells der industriellen Beziehungen. Es lassen sich also gute Gründe dafür anführen, Internationalisierung auf unternehmensspezifischer Ebene zu messen. Vor diesem Hintergrund konzentrieren sich die weiteren Überlegungen dieses Beitrags auf die Messung der Internationalisierung von Unternehmen.

1 Für einen Überblick siehe auch Dörrenbächer (2000). 


\section{Ein- oder Mehrdimensionalität von Internationalisierung?}

Die Zahl der potenziellen Indikatoren zur Messung von Internationalisierung ist groß. In einer Übersicht haben Germann, Rürup und Setzer 47 Indikatoren identifiziert, von denen sich allein 30 zur Messung von unternehmensspezifischen Internationalisierungsgraden verwenden lassen (Germann et al. 1995; Rürup und Setzer 1996). 30 unterschiedliche Möglichkeiten der Operationalisierung sind letztlich ebenso viele Antworten auf die Frage, wie internationalisiert ein Unternehmen ist. Wie lässt sich Ordnung in eine solche Vielfalt bringen?

Eine Möglichkeit besteht darin, sich für einen einzelnen Indikator zu entscheiden, den man als das wichtigste Merkmal der Internationalisierung definiert hat. Alle anderen Operationalisierungsmöglichkeiten bleiben dabei unbeachtet. Martin Glaum z.B. definiert Internationalisierung anhand der Produktion (Glaum 1996). Dieses Verfahren besticht zwar durch seine Eindeutigkeit, wird dem Stand der Internationalisierungsdebatte jedoch nicht gerecht. Es gibt Anzeichen dafür, dass sich die Formen der Internationalisierung unterscheiden und verändern können. Johanson und Vahlne beschreiben ein Phasenmodell, in dem ein Unternehmen seine internationalen Aktivitäten mit dem Export beginnt. Es folgt die Errichtung von Vertriebs-, Lager- und Serviceeinrichtungen sowie Lizenzvergabe und Franchising. Schließlich folgen eigene Produktionsstätten, die in einer letzten Stufe zu eigenständigen Tochtergesellschaften werden können (Johanson und Vahlne 1977; Dülfer 1999). Ein stagnierender ausländischer Anteil an Umsatz oder Beschäftigten muss daher nicht notwendigerweise bedeuten, dass die Internationalisierung des Unternehmens insgesamt stagniert. Es kann sich auch lediglich die Form verändert haben. Ein einzelner Internationalisierungsindikator kann also zu falschen Ergebnissen führen. Zudem liefert die von Germann, Rürup und Setzer beschriebene Anzahl möglicher Indikatoren einen Hinweis darauf, dass Internationalisierung als multidimensionales Phänomen betrachtet werden sollte.

Vor diesem Hintergrund muss man sich der Zusammenfassung mehrerer Indikatoren zuwenden. In den Sozialwissenschaften stehen dafür zwei Verfahren zur Verfügung: die Konstruktion von Typologien oder Indizes.

\section{Typologien und Indizes}

Eine Typologie bildet die Gesamtheit aller verschiedenen Kombinationen der zu Grunde liegenden Indikatoren $\mathrm{ab}$ und bildet äquivalente Kombinationsklassen. Ein Index fasst hingegen einige Kombinationen zu neuen Klassen zusammen, wobei auch eine Ordnungsrelation definiert wird. Die Vorteile eines Index bestehen darin, dass Informationen verdichtet werden, da der Merkmalsraum um die Typen reduziert wird, die nicht besetzt sind. Dadurch lassen sich höhere Fallzahlen mit einer größeren Anzahl an Variablen und ihren Ausprägungen verarbeiten. Zudem können aus der hergestellten Ordnungsrelation Verhältnisaussagen abgeleitet werden. Während Typologien darauf abzielen, Unterschiede zu beschreiben (Typ A ist anders als Typ B), ermöglichen Indizes die Herstellung von Relationen und Rangordnungen zwischen Typen. 
a) Internationalisierungstypologien. In der Debatte über die Internationalisierung von Unternehmen spielt die Typologie von Bartlett und Ghoshal eine bedeutende Rolle (Bartlett und Ghoshal 1989). Durch Fallstudien über die Internationalisierung von neun Unternehmen aus jeweils drei Branchen und Nationen entwickeln sie vier Klassen. Multinationale Unternehmen zeichnen sich demnach aus durch eine länderspezifische und dezentralisierte Strategie, bei der Produkte an die lokalen Bedingungen angepasst werden und die Niederlassungen in den jeweiligen Ländern über ein relativ großes Maß an Selbständigkeit verfügen. Demgegenüber grenzt sich das globale Unternehmen $\mathrm{ab}$, das sich durch ein hohes $\mathrm{Ma}$ an Zentralisierung auszeichnet, wobei relativ einheitliche Produkte weltweit exportiert werden. Mit dem internationalen Unternehmen sowie dem transnationalen Unternehmen werden zusätzlich zwei Mischtypen vorgeschlagen (Bartlett und Goshal 1986, 1987a, 1987b, 1989). Die Typologie stößt in den soziologischen Untersuchungen über die Internationalisierung von Unternehmen auf große Resonanz (z.B. Lane 1998; Hirsch-Kreinsen 1992). Die Merkmalsunterschiede zwischen den Klassen erscheinen jedoch ungenau. Dies gilt besonders für die beiden Mischtypen. Auch ist nicht klar, an welchen Punkten jeweils Typensprünge identifiziert werden können, wann also ein Unternehmen z.B. nicht mehr als multinational, sondern als transnational einzustufen ist. Die Grenzziehung zwischen den einzelnen Klassen scheint aber notwendig zu sein, da man bei der Untersuchung größerer Samples kaum erwarten kann, dass sich die Unternehmen fein säuberlich in die konstruierten vier Typen gruppieren. In der Internationalisierungsdebatte wird die Unklarheit der Typen vor allem daran deutlich, dass verschiedene Autoren den gleichen Begriffen unterschiedliche Inhalte zuordnen. So stellt das multinationale Unternehmen für Bartlett den Typ mit der stärksten Dezentralisierungsausprägung dar (Bartlett 1989: 431). Christel Lane weist diesen Platz hingegen dem transnationalen Unternehmen zu (Lane 1998: 464). Gestützt wird die Kritik durch eine empirische Untersuchung von Leong und Tan, die anhand eines Samples von 131 weltweit aktiven Konzernen u.a. den empirischen Gehalt der einzelnen Typen testeten (Leong und Tan 1993). Sie kommen zu dem Ergebnis, dass sich die beiden Extremfälle, das globale sowie das multinationale Unternehmen, relativ klar empirisch herauskristallisieren. Die beiden Mischtypen sind jedoch nur schwer zu unterscheiden. Ein differenziertes und differenzierendes Abbild der Internationalisierung von Unternehmen erhält man durch diese Typologie also nicht.

b) Internationalisierungsindizes. Eine zweite Möglichkeit der Zusammenfassung von Indikatoren besteht in der Konstruktion von Indizes. Die Verknüpfung muss dabei theoretisch wie empirisch begründbar sein. Diese Prämisse wird jedoch nicht immer eingehalten. Bisher wurden in der Debatte drei verschiedene Indizes vorgeschlagen: der Transnationalitätsindex der UNCTAD (z.B. UNCTAD 1999), der Index von Daniel Sullivan (Sullivan 1994) sowie der Index von Grazia Ietto-Gillies (Ietto-Gillies 1998).

Die UNCTAD lässt drei Variablen mit jeweils gleichem Gewicht in ihren Index einfließen: den ausländischen Anteil an den Vermögenswerten, am Umsatz sowie an der Beschäftigung. In ihrem jährlichen World Investment Report weist sie ihren Index einschließlich der zu Grunde liegenden Rohdaten für die weltweit hundert größten multinationalen Konzerne (gemessen an den absoluten ausländischen Vermögenswerten) aus. Eine eigene Berechnung ergab, dass sich die ausländischen Vermögenswerte 
sowie der Auslandsumsatz zu einem gemeinsamen Faktor bündeln lassen, mit dem der Beschäftigungsindikator empirisch jedoch nicht einher geht. Dieses kann durch die im internationalen Vergleich variierende Kapitalausstattung pro Beschäftigtem begründet sein. Zudem setzt sich das Sample der UNCTAD aus Unternehmen sowohl aus großen als auch aus kleinen Staaten zusammen. Es gibt einen Zusammenhang zwischen der Größe des Basislandes und der Internationalität eines Unternehmens: Schweizer Unternehmen internationalisieren sich auf Grund des kleinen Binnenmarktes schneller als amerikanische. Vor diesem Hintergrund erstaunt es nicht, wenn die Spitzenplätze im Ranking der UNCTAD vorrangig von Unternehmen aus kleinen Industriestaaten eingenommen werden (Ietto-Gillies 1998; UNCTAD 1998). Das spricht für die Auswahl und Untersuchung nationaler Samples. Während die Datensammlungen der UNCTAD also sehr hilfreich sind, kann die Verknüpfung der Indikatoren zu einem Index hingegen weder empirisch noch theoretisch überzeugend begründet werden.

Der Internationalisierungsindex von Grazia Ietto-Gillies nutzt die Daten der UNCTAD, um auf dieser Grundlage das Problem der regionalen Streuung zu thematisieren. Sie argumentiert, die Dichotomie zwischen national und international werde der Komplexität des Phänomens nicht gerecht, und plädiert für einen Indikator, der den Grad an regionaler Konzentration oder Streuung berücksichtigt. Ein hoher Grad an regionaler Streuung weise darauf hin, dass Standortvorteile optimal genutzt werden können. Zudem könne er die Fragmentierung der Arbeitnehmer befördern und somit ihre Verhandlungsposition schwächen (Ietto-Gillies 1998: 23). Die Streuung der Auslandsaktivitäten erscheint auch als ein Indikator für die Intensität von Internationalisierung, $\mathrm{da}$ in einem Unternehmen mit Niederlassungen in 100 Staaten eine intensivere Vermittlung zwischen unterschiedlichen Kulturen notwendig wird als in einem Unternehmen, das nur in zwei Ländern vertreten ist. Ietto-Gillies konstruiert einen Streuungsindex (Network-Spread-Index), den sie mit dem Transnationalitätsindex multipliziert, um einen allgemeinen Internationalisierungsindex zu erhalten. So bestechend die inhaltliche Begründung für die regionale Streuung als Indikator für die Internationalisierung ist, so wenig überzeugt doch das Verfahren, Streuungs- und UNCTAD-Index zu verknüpfen, da der Rangkorrelationskoeffizient lediglich bei 0,4 liegt (Ietto-Gillies 1998: 35). Die empirische Kohärenz erweist sich als eher schwach, sodass man das Streuungsmaß ebenso als eigenständigen Indikator ansehen könnte. Unabhängig davon wird die Arbeit von Ietto-Gillies zusätzlich von den Problemen beeinträchtigt, die in Bezug auf länderübergreifende Samples schon beim UNCTAD-Index diskutiert wurden. Der Ietto-Gillies-Index auf Grundlage des UNCTAD-Samples kann deshalb nicht vollständig überzeugen.

Daniel Sullivan konstruiert einen dritten Index anhand eines Samples aus 74 Unternehmen. Diese entstammen der Rangliste der 100 internationalisiertesten amerikanischen Unternehmen, gemessen an der Höhe des Auslandsumsatzes. Sullivan sichert seinen Index faktorenanalytisch ab. Er erhebt den Anteil an nichtamerikanischen Tochtergesellschaften und den ausländischen Anteil an den Vermögenswerten sowie am Auslandsumsatz (Sullivan 1994). Zudem fügt er seinem Index zwei kulturelle Indikatoren hinzu. Die Auslandserfahrung des Top-Managements stellt einen Indikator dar für internationalisierte Handlungsorientierungen und Managementkulturen im Unternehmen. Dabei greift Sullivan auf ältere konzeptionelle Arbeiten zurück (Perlmutter 1969; 
Maisonrouge 1983). Zudem erhebt er die psychische Streuung von Auslandsaktivitäten. Dahinter verbirgt sich die Annahme, der Internationalisierungsgrad eines Unternehmens steige mit zunehmenden Aktivitäten in weit voneinander entfernten Ländern, da geographische sowie kulturelle Distanzen überbrückt werden müssten. Ein Unternehmen mit Produktionsstätten in den USA und Ägypten sei demnach internationalisierter als ein Unternehmen mit Niederlassungen in den USA und Kanada (Johanson und Vahlne 1977; Sullivan und Bauerschmidt 1990). In diesem Zusammenhang wird auf Arbeiten zurückgegriffen, die die Welt in psychische oder kognitive Zonen einteilen (Ronen und Shenkar 1985; Hofstede 1993). Es ist kritisiert worden, dass die verknüpften Indikatoren auf unterschiedlichen Ebenen angesiedelt sind. Sullivan gießt Handlungsorientierungen, Struktureigenschaften und ein Performanzkriterium in einen gemeinsamen Index (Ramaswamy et al. 1996: 174). Es ist jedoch unbestreitbar Sullivans Verdienst, Indikatoren in die empirische Internationalisierungsdebatte einzuführen, die darauf hinweisen, dass Unternehmen nicht nur als wirtschaftlich-technische Institution verstanden werden, sondern auch als soziales Gebilde, die durch ihre Herrschaftsstruktur, den Grad und die Form funktionaler Organisation sowie durch ihre Unternehmenskulturen gekennzeichnet sind. Diese Perspektive scheint bei der Messung von Internationalisierung bisher noch unterbeleuchtet zu sein.

Als Resultat der Betrachtung vergangener Messversuche lässt sich festhalten, dass eine Reihe viel versprechender Indikatoren entwickelt wurde. Die Indexkonstruktion überzeugte bisher jedoch nur wenig, da ihre theoretische und empirische Kohärenz häufig fragwürdig bleibt. Zudem hat sich herausgestellt, dass die Messung von Internationalisierung immer kontextabhängig ist. Nationale Samples scheinen dabei gegenüber internationalen Samples deutliche Vorteile aufzuweisen.

\section{Die realwirtschaftliche und die kapitalmarktbezogene Dimension der Internationalisierung}

Die vorangestellten methodischen Erwägungen haben unsere eigene empirische Untersuchung beeinflusst. Die bereits erwähnten Vorteile nationaler Samples veranlassten uns dazu, ein Messverfahren zu entwickeln, das die Internationalisierung deutscher Unternehmen abbildet. Auf der Basis unserer konzeptionellen Überlegungen sowie der empirischen Ergebnisse schlagen wir vor, zwischen der realwirtschaftlichen und der $k a-$ pitalmarktbezogenen Internationalisierung von Unternehmen zu unterscheiden. Dabei halten wir eine Übertragbarkeit unserer Aussagen von deutschen auf kontinentaleuropäische Unternehmen für wahrscheinlich.

Die realwirtschaftliche Dimension beschreibt die physische, d.h. die produktionsund güterwirtschaftliche Ausbreitung der Unternehmen. Ausgehend von der Einschätzung, dass die sichtbaren grenzüberschreitenden Aktivitäten das wichtigste Merkmal der Internationalisierung von Unternehmen darstellen, ist hier auch ein Großteil der empirischen Forschung angesiedelt. Fast alle Studien konzentrieren sich auf die ausländischen Anteile an Produktion und Absatz, insbesondere an Beschäftigung, Investitionen und Absatz (z.B. UNCTAD 1997; Riahi-Belkaoui 1998; Glaum 1996; Döhrn 1997). 
Die kapitalmarktbezogene Internationalisierung beschreibt die Ausrichtung der Unternehmen auf internationale Kapitalmärkte. Sie ist bisher in der Forschung kaum berücksichtigt worden. Es spricht jedoch vieles dafür, dass diese Dimension wichtige Aspekte der Internationalisierung abbildet, die durch realwirtschaftliche Aktivitäten nicht erfasst werden. Die Forschung über Corporate Governance hat herausgearbeitet, dass im internationalen Vergleich Eigentümerstruktur und Unternehmensfinanzierung durch institutionelle Einflüsse erheblich variieren (Pedersen und Thomsen 1997; Porta et al. 1998). Dabei wird zwischen einem anglo-amerikanischen und einem kontinentaleuropäischen Modell unterschieden. Letzteres zeichnet sich durch ein geringes $\mathrm{Maß}$ an Börsenfinanzierung, einen geringen Anteil an Aktienstreubesitz, ein hohes $\mathrm{Maß}$ an Bankeneinfluss sowie durch die faktische Abwesenheit feindlicher Übernahmen aus. Insbesondere in Deutschland kommt Unternehmenskontrolle weniger über die Kapitalmärkte, als vielmehr über die direkt am Unternehmen beteiligten Gruppen (Insider) zustande.

Das europäische Corporate-Governance-Modell ist in Bewegung. In den 90er Jahren beginnen kontinentaleuropäische Unternehmen, sich anglo-amerikanischen Gepflogenheiten anzupassen. Dazu gehört die Zunahme der Notierungen an ausländischen Börsen sowie die Übernahme internationaler Rechnungslegungsstandards. Mit diesen Maßnahmen wird der Zugang internationaler Investoren zu den Unternehmen erleichtert. Im Zuge dessen ist in zunehmendem Maße eine Orientierung an der ShareholderValue-Strategie zu beobachten, die darauf abzielt, den Wert eines Unternehmens für (internationale) Aktionäre zu erhöhen. Die Dimension der Orientierung an den Kapitalmärkten weist auch mit Blick auf die zu erwartenden Folgen eine eigene Qualität auf, da die Handlungen der am Unternehmen beteiligten Gruppen (Insider) nun stärker durch renditeorientierte Aktionäre (Outsider) kontrolliert werden. Darüber hinaus kann zwar die Orientierung an internationalen Kapitalmärkten aktiv von den Unternehmen vorangetrieben werden, inwieweit sich diese aber auch in einer internationalen Eigentümerstruktur niederschlägt, kann vom Unternehmen nur bedingt beeinflusst werden.

Realwirtschaftliche und kapitalmarktbezogene Internationalisierung sind also grundsätzlich voneinander zu unterscheiden. Realwirtschaftliche Internationalisierung ist die räumliche Streuung von Produktion und Absatz über Grenzen hinweg. Unter kapitalmarktbezogener Internationalisierung wird eine Anpassung an anglo-amerikanische Managementstandards verstanden, die auf Kommunikation mit den Kapitalmarktteilnehmern setzt, anstatt sich von diesen abzuschotten.

\section{Projektdesign}

Anhand einer empirischen Untersuchung wollen wir den Nachweis erbringen, dass sich zwei Dimensionen der Internationalisierung von deutschen und kontinentaleuropäischen Unternehmen nicht nur theoretisch plausibel begründen, sondern auch empirisch bestätigen lassen. ${ }^{2}$

2 Für eine ausführlichere Darstellung des empirischen Materials siehe Hassel et al. (2000). 


\section{Auswahl der untersuchten Unternehmen}

Die Untersuchung wird anhand der 100 größten deutschen Unternehmen durchgeführt. Grundlage ist das Ranking aus dem aktuellen Gutachten der Monopolkommission (1998). Die Größe wird dabei nicht am Umsatz, sondern an der Wertschöpfung des Unternehmens gemessen. Dieses Vorgehen birgt mehrere Vorteile. Erstens erlaubt die Wertschöpfung die Einbeziehung von Unternehmen des Kredit- und Versicherungswesens in die Untersuchung. Zweitens werden die Effekte unterschiedlicher Preisentwicklungen in einzelnen Branchen ausgeschaltet. Zudem ist die Wertschöpfung besser geeignet, die vertikale Integration verschiedener Branchen darzustellen. So ist z.B. bei Handelsunternehmen, die nicht oder nur in unbedeutendem Umfang vertikal integriert sind, das Verhältnis zwischen Wertschöpfung und Umsatz regelmäßig geringer als bei anderen Unternehmen (Monopolkommission 1998: 153).

Andere Untersuchungen wählten ihre Samples nach der Höhe des Auslandsumsatzes aus (Sullivan 1994; Stopford und Dunning 1983; Daniels und Bracker 1989), sodass ausschließlich stark internationalisierte Unternehmen in das Blickfeld geraten. Durch die Größe als Auswahlkriterium für unsere Untersuchung erwarten wir eine größere Varianz zwischen stark und weniger stark internationalisierten Unternehmen, vor allem durch die ehemaligen Staatsbetriebe, die erst in den 90er Jahren privatisiert wurden. Gleichzeitig decken wir mit unserer Auswahl einen hohen Anteil an unternehmerischen ausländischen Aktivitäten ab. Die 100 Unternehmen beschäftigen knapp ein Drittel aller Auslandsbeschäftigten deutscher Unternehmen. Innerhalb der deutschen Grenzen beschäftigen sie knapp 16 Prozent aller im privaten Sektor tätigen Arbeitnehmer, die einen Wertschöpfungsanteil von fast 18 Prozent erwirtschaften. In unserem Sample sind 64 Industrieunternehmen vertreten, die sich aus den Sektoren Chemie (11), Maschinenbau (10), Automobil (8) und anderen zusammensetzen. Die restlichen 36 Unternehmen sind im Dienstleistungsbereich tätig. Sie setzen sich aus Banken (10), Versicherungen (8), Handel (10) und anderen zusammen.

\section{Indikatoren}

Die beiden Internationalisierungsdimensionen werden anhand von sechs Indikatoren operationalisiert. Die Daten beziehen sich auf das Jahr 1996, im Falle der kapitalmarktbezogenen Dimension sind die Bezugsjahre 1996-1999. Drei Indikatoren beziehen sich auf die realwirtschaftliche Dimension. Der ausländische Anteil am Gesamtumsatz (AGU) ist der gängigste Internationalisierungsindikator (Sullivan 1994). Er ist auch Bestandteil aller bereits genannten Indizes (Sullivan 1994; UNCTAD 1997; Ietto-Gillies 1998). Ähnlich geläufig ist der ausländische Anteil an der Gesamtbeschäftigung (AGB). Zwei der Indizes arbeiten mit dieser Variable (UNCTAD 1997; IettoGillies 1998). Der dritte unserer Indikatoren bezieht sich auf die Arbeit von IettoGillies und beschreibt die regionale Streuung der Auslandsaktivitäten (STREU). Sie wird anhand der Zahl der Länder, in denen ein Unternehmen Beteiligungen hält, gemessen. Dabei haben wir uns dafür entschieden, alle Beteiligungen zu berücksichtigen, unabhängig von ihrer Größe oder von ihrem Status als verbundene, assoziierte oder konsoli- 
dierte Tochter- oder Enkelunternehmen. Eine Schwierigkeit bei der Erhebung liegt in der schlechten Datenqualität, denn die Veröffentlichungspraxis variiert zwischen den Unternehmen erheblich. Einige Unternehmen mit offensichtlich sehr vielen Beteiligungen zählen in ihren Geschäftsberichten nur sehr wenige beispielhaft auf, während andere mit sehr wenigen Beteiligungen dazu tendieren, jede Kleinstbeteiligung zu nennen. Um die schlechte Datenqualität auszugleichen, haben wir bei der Erhebung auch andere Informationsquellen genutzt und lediglich eine Grobklassifikation der Unternehmen vorgenommen. Wir unterscheiden zwischen starker, mittlerer und schwacher Streuung.

Die kapitalmarktbezogene Internationalisierungsdimension wird anhand von drei Indikatoren abgebildet. Der ausländische Anteil am Aktienbesitz (AAB) beschreibt die aktuelle Nähe zu internationalen Kapitalmarktteilnehmern, nämlich die Internationalisierung der Eigentümerstruktur. Zwei weitere Indikatoren beziehen sich auf die Öffnung gegenüber internationalen Kapitalmärkten. Dabei handelt es sich um die Zahl der Notierungen an ausländischen Börsen (NAB) sowie um die gewählten Bilanzierungsstandards (BS). Wenn Unternehmen nicht nach dem deutschen HGB bilanzieren, sondern nach internationalen Regeln (International Accounting Standards (IAS) oder US General Accepted Accounting Principles (US-GAAP)), erhöht dieses auch die Attraktivität einer Investition für internationale Anleger, und es erleichtert die Kommunikation auf internationalem Parkett.

Bei der Operationalisierung der kapitalmarktbezogenen Dimension wird die Kontext- und Sampleabhängigkeit eines Messverfahrens besonders deutlich. Da wir davon ausgehen, dass der Prozess beschrieben werden kann als eine Annäherung deutscher und kontinentaleuropäischer Praktiken an anglo-amerikanische Standards, könnte die Operationalisierung in dieser Form nicht beliebig übertragen werden.

\section{Datenquellen}

Datenquellen für den ausländischen Anteil an Umsatz und Beschäftigung waren die Berichte der Monopolkommission, nicht veröffentlichte Daten der Monopolkommission sowie eine Zusammenstellung der Forschungsgemeinschaft FAST e.V. in Berlin im Auftrag des Max-Planck-Instituts für Gellschaftsforschung. Im letzteren Fall wurden Geschäftsberichte sowie zusätzliche Informationen (Presseerklärungen, Unternehmensbroschüren etc.) ausgewertet. Zudem wurde auf Daten zurückgegriffen, die im Rahmen des Projekts Globalisierung und internationale Mobilität deutscher Industrieunternehmen erhoben wurden (Wortmann et al. 1997). Die regionale Streuung wurde anhand der Geschäftsberichte sowie aus zusätzlichen Unternehmensbroschüren erhoben. Die Internationalisierung der Eigentümerstruktur setzt sich aus dem internationalen Einzelbesitz sowie dem internationalen Anteil am Streubesitz zusammen. Datenquelle für den Einzelbesitz war das Gutachten der Monopolkommission aus dem Jahr 1998. Die ausländischen Anteile beim Streubesitz wurden mittels Presse-, Geschäftsberichtsund Internetrecherchen sowie durch Anfragen an die Investor-Relations-Abteilungen der Unternehmen erhoben. Die Anzahl ausländischer Börsennotierungen geht aus der OnVista-Datenbank hervor. Die Angaben zu den Bilanzierungsmethoden stammen aus Geschäftsberichten und Pressemitteilungen. 


\section{Datenanalyse}

Für die Datenanalyse wurden 14 der 100 Unternehmen ausgeschlossen, da sie Tochterfirmen ausländischer Unternehmen sind. Diese Sonderfälle würden die Ergebnisse verzerren, da Töchter ausländischer Konzerne in der Regel selbst wenig Auslandsaktivitäten zeigen, während sie zu 100 Prozent in ausländischem Besitz sind. Für die verbleibenden 86 Unternehmen konnten für 79 Firmen die Daten für die realwirtschaftliche und für 68 Firmen die Daten für die kapitalmarktbezogene Dimension vollständig erhoben werden. Die vorhandenen Lücken sind überwiegend auf fehlende Angaben für die geographische Streuung und den Anteil ausländischer Eigentümer zurückzuführen. Wie auf Grund unserer Unternehmensauswahl schon vermutet, weist ein Teil der Unternehmen (9 Prozent) keinerlei Anzeichen realwirtschaftlicher Internationalisierung auf. 33 Firmen (38 Prozent) zeigen keine Form der Orientierung an internationalen Kapitalmärkten.

Zur Bestätigung unserer Annahme, dass die von uns gewählten Indikatoren zwei unterschiedliche Dimensionen der Internationalisierung abbilden, wurde zunächst die Korrelationsmatrix der sechs Variablen errechnet (vgl. Tabelle 1).

Tabelle 1: Korrelationsmatrix der Indikatoren (Pearson)

\begin{tabular}{lcccccc}
\hline & AGB & AGU & STREU & BS & NAB & AAB \\
\hline AGB & 1.00 & $.725^{* *}$ & $.679^{* * 1}$ & $.260^{* 1}$ & $.295^{* *}$ & $.265^{*}$ \\
AGU & & 1.00 & $.656^{* * 1}$ & $.315^{* * 1}$ & $.346^{* *}$ & $.365^{* *}$ \\
STREU & & & 1.00 & $.329^{* * 1}$ & $.318^{* * 1}$ & $.306^{* 1}$ \\
BS & & & 1.00 & $.629^{* * 1}$ & $.784^{* * 1}$ \\
NAB & & & & & 1.00 & $.589^{* *}$ \\
AAB & & & & & & 1.00 \\
\hline
\end{tabular}

** Die Korrelation ist auf dem Niveau von 0,01 (2-seitig) signifikant.

* Die Korrelation ist auf dem Niveau von 0,05 (2-seitig) signifikant.

1 Spearman-Rho

Sowohl die drei Indikatoren der realwirtschaftlichen Dimension (AGB, AGU, STREU) als auch die drei Indikatoren der kapitalmarktbezogenen Dimension (BS, NAB, AAB) korrelieren jeweils untereinander hoch, während alle anderen Koeffizienten zwar auch positiv, jedoch deutlich niedriger ausfallen.

Die abschließende Faktorenanalyse ${ }^{3}$ führt - wie es die bisherigen Ergebnisse schon vermuten lassen - zur Extraktion von zwei Faktoren, denen die Variablen gemäß unserer theoretischen Überlegungen zugeordnet sind (vgl. Tabelle 2).

Wir erhalten zwei Indizes, indem wir den Durchschnitt der jeweils drei z-transformierten Variablen berechnen, ohne eine Gewichtung vorzunehmen.

$\mathrm{REAL}=(\mathrm{zAGU}+\mathrm{zAGB}+\mathrm{zSTREU}) / 3$

$\mathrm{KAPITAL}=(\mathrm{zAAB}+\mathrm{zBS}+\mathrm{zNAB}) / 3$

3 Idealerweise werden in eine Faktorenanalyse nur intervallskalierte Variablen einbezogen. In diesem Fall werden die ordinalen Daten von uns als metrisch interpretiert. 
Tabelle 2: Rotierte Komponentenmatrix ${ }^{\mathrm{a}}$

\begin{tabular}{|c|c|c|c|}
\hline & \multicolumn{2}{|c|}{ Komponente } & \multirow[t]{2}{*}{ Communality } \\
\hline & 1 & 2 & \\
\hline AGB & .906 & .100 & .831 \\
\hline AGU & .866 & .207 & .793 \\
\hline STREU & .813 & .254 & .726 \\
\hline BS & .112 & .880 & .787 \\
\hline NAB & .240 & .733 & .595 \\
\hline $\mathrm{AAB}$ & .188 & .903 & .851 \\
\hline
\end{tabular}

Extraktionsmethode: Hauptkomponentenanalyse. Rotationsmethode: Varimax mit Kaiser-Normalisierung.

a Die Rotation ist in 8 Iterationen konvergiert.

Tabelle 3: Korrelationsmatrix der Indikatoren und Indizes

\begin{tabular}{lllllll}
\hline & AGB & AGU & STREU & BS & NAB & AAB \\
\hline REAL & $.901^{* *}$ & $.894^{* *}$ & $.838^{* *}$ & & & \\
KAPITAL & & & & $.899^{* *}$ & $.823^{* *}$ & $.899^{* *}$ \\
\hline
\end{tabular}

** Die Korrelation ist auf dem Niveau von 0,01 (2-seitig) signifikant.

Bei beiden Indizes sind die Korrelationen zwischen ihnen und ihren konstituierenden Variablen sehr hoch (vgl. Tabelle 3).

Die Ranglistenplätze der Unternehmen auf den einzelnen Skalen sind für die jeweils 25 hochrangigsten Unternehmen gemäß den beiden Indizes REAL und KAPITAL in den Tabellen 4 und 5 aufgeführt.

Im Gegensatz zur hohen empirischen Kohärenz innerhalb der beiden Indizes REAL und FINANZ - fällt der statistische Zusammenhang zwischen ihnen deutlich geringer aus. Der Rangkorrelationskoeffizient beträgt $r=0,41$ und bestätigt somit unsere Annahme, dass mit diesen Indizes zwei verschiedene Dimensionen der Internationalisierung erfasst werden.

Unter den 86 von uns untersuchten Unternehmen befinden sich 18 Firmen, die keine Aktiengesellschaften sind. Man könnte argumentieren, dass diesen auf Grund ihrer Rechtsform der Zugang zu internationalen Kapitalmärkten erschwert wird. Um einen möglichen Rechtsform-Effekt auszuschließen, wurden die statistischen Analysen noch einmal für die Gesamtheit aller Aktiengesellschaften wiederholt. Unter Ausschluss der Unternehmen, deren Zugang zu internationalen Kapitalmärkten eingeschränkt ist, während es keinerlei Hindernisse in Bezug auf die realwirtschaftliche Internationalisierung gibt, erhöht sich der Korrelationskoeffizient erwartungsgemäß $(r=0,60, N=49)$. Die Faktorenanalyse führt jedoch zu den gleichen und ebenso eindeutigen Resultaten wie unter Einbezug aller 86 Unternehmen. Somit kann die Unterscheidung von zwei Internationalisierungsdimensionen selbst unter Anwendung strengerer Maßstäbe aufrecht erhalten werden. 
Tabelle 4: Rangliste: Realwirtschaftliche Internationalisierungsdimension (TOP 25 von 86 Unternehmen)

\begin{tabular}{lccrc}
\hline Unternehmen & REAL & STREU & AGU & AGB \\
\hline Boehringer Sohn C.H. & 1 & Stark & 4 & 3 \\
Hoechst AG & 2 & Stark & 3 & 5 \\
Henkel KG & 3 & Stark & 8 & 2 \\
Schering AG & 4 & Stark & 1 & 11 \\
Bayer AG & 5 & Stark & 2 & 13 \\
Franz Haniel \& Cie. GmbH & 6 & Stark & 13 & 4 \\
SAP AG & 7 & Stark & 6 & 15 \\
Beiersdorf AG & 8 & Stark & 19 & 6 \\
Bertelsmann AG & 9 & Stark & 17 & 8 \\
Freudenberg \& Co. KG & 10 & Stark & 15 & 10 \\
BMW AG & 11 & Stark & 9 & 22 \\
BASF AG & 12 & Stark & 7 & 28 \\
Bosch, Robert GmbH & 13 & Stark & 24 & 18 \\
Siemens AG & 14 & Stark & 23 & 21 \\
Allianz AG & 15 & Stark & 29 & 12 \\
Linde AG & 16 & Stark & 21 & 24 \\
Bosch-Siemens Hausgeraete GmbH & 17 & Stark & 27 & 23 \\
Carl-Zeiss-Stiftung & 18 & Stark & 10 & 33 \\
Bilfinger + Berger Bau-AG & 19 & Mittel & 36 & 1 \\
Continental AG & 20 & Mittel & 14 & 9 \\
Mannesmann AG & 21 & Stark & 26 & 29 \\
Metallgesellschaft AG & 22 & Stark & 11 & 42 \\
Degussa AG & 23 & Mittel & 5 & 27 \\
Daimler-Benz AG & 24 & Stark & 22 & 38 \\
Wacker-Chemie GmbH & 25 & Stark & 16 & 45 \\
\hline
\end{tabular}

\section{Die Internationalisierung deutscher Großunternehmen}

Die Varianz zwischen den Unternehmen und Sektoren ist bei allen Indikatoren und Dimensionen groß. Dieser Sachverhalt stützt den methodischen Ausgangspunkt, bei der empirischen Betrachtung von Internationalisierungsprozessen auf der Unternehmensebene anzusetzen, weil aggregierte Analysen diese Varianz verdecken würden. Gleichzeitig wird die Aussage unterstrichen, dass es sich bei der Internationalisierung nicht um ein universelles Phänomen handelt.

\section{Realwirtschaftliche Dimension}

Die güterwirtschaftliche Seite der Internationalisierung ist am weitesten fortgeschritten. Mehr als ein Drittel aller von den 100 größten deutschen Unternehmen produzierten Güter und Dienstleistungen werden im Ausland abgesetzt. Auslandsanteile am Gesamtumsatz von über 70 Prozent sind nicht selten. Das gilt beispielsweise für die meisten großen Unternehmen der chemischen Industrie, aber auch für die SAP AG und die BMW AG. Die Schering AG erzielt mit einem Anteil von 85 Prozent den Spitzenwert. 
Tabelle 5: Rangliste: Kapitalmarktbezogene Internationalisierungsdimension (TOP 25 von 86 Unternehmen)

\begin{tabular}{lcccr}
\hline Unternehmen & Kapital & BS & NAB & AAB \\
\hline Bayer AG & 1 & IAS & 1 & 4 \\
Hoechst AG & 2 & IAS & 3 & 3 \\
Deutsche Bank AG & 3 & IAS & 4 & 7 \\
Daimler-Benz AG & 4 & US-GAAP & 6 & 10 \\
Mannesmann AG & 5 & IAS & 11 & 2 \\
Dresdner Bank AG & 6 & IAS & 5 & 17 \\
Siemens AG & 7 & US-GAAP & 8 & 8 \\
BASF AG & 8 & US-GAAP & 6 & 14 \\
VEBA AG & 9 & US-GAAP & 9 & 6 \\
Metallgesellschaft AG & 10 & US-GAAP & 27 & 1 \\
Deutsche Telekom AG & 11 & US-GAAP & 11 & 5 \\
Schering AG & 12 & IAS & 13 & 7 \\
BMW AG & 13 & IAS & 13 & 12 \\
Commerzbank AG & 14 & IAS & 27 & 8 \\
VIAG AG & 15 & IAS & 13 & 21 \\
RWE AG & 16 & IAS & 13 & 23 \\
Allianz AG & 17 & IAS & 13 & 25 \\
Linde AG & 18 & IAS & 27 & 13 \\
Thyssen AG & 19 & US-GAAP & 13 & 26 \\
Metro Holding AG & 20 & IAS & 21 & 20 \\
Deutsche Lufthansa AG & 21 & IAS & 27 & 15 \\
MAN AG & 22 & IAS & 13 & 27 \\
Degussa AG & 23 & US-GAAP & 21 & 26 \\
Preussag AG & 24 & IAS & 27 & 19 \\
Münchener Rueckversicherungsgesellschaft AG & 25 & IAS & 27 & 22 \\
\hline
\end{tabular}

Die Unternehmen ohne Auslandsumsatz sind vor allem in der Energieversorgung sowie im Dienstleistungsbereich tätig.

Auch die Internationalisierung der Produktion hat ein hohes Maß erreicht. Der durchschnittliche Anteil der Auslandsbeschäftigung an der Gesamtbeschäftigung liegt 1996 bei knapp 28 Prozent. Die Bilfinger + Berger AG besetzt hier mit 73 Prozent die Spitzenposition. Unter den ersten zehn Unternehmen ist dies jedoch der einzige Baukonzern. Die Unternehmen der Chemieindustrie stellen auch hier die größte Gruppe. Etwa jedes fünfte Unternehmen unseres Samples verfügt über keine Auslandsbeschäftigung. Wie schon beim Umsatz wird auch diese Gruppe im Wesentlichen durch Energieversorgungs- und Dienstleistungsunternehmen, die auf den Binnenmarkt ausgerichtet sind (z.B. Flughafen Frankfurt AG, Axel Springer Verlag AG), gebildet.

In Bezug auf die regionale Streuung der Aktivitäten ist festzuhalten, dass kaum ein Unternehmen mehr darauf verzichtet, im Ausland präsent zu sein. Fünf Unternehmen halten keine Beteiligungen im Ausland. ${ }^{4}$ Spitzenreiter der Skala sind die Bertelsmann AG, die Beiersdorf AG sowie die Mannesmann AG mit Beteiligungen in 45 Ländern.

4 Es handelt sich um AVA, Kaiser's Kaffee-Geschäft AG, Metro AG, Philip Morris GmbH und Veag AG. Dies bedeutet jedoch nicht, dass die Unternehmen nicht im Ausland präsent sind. So hat die Metro Betriebsstätten in 19 Ländern. 
Die Hälfte der Unternehmen geben maximal 13 Länder mit Auslandsbeteiligungen an. Das Unternehmen mit der höchsten Präsenz in Europa ist die Bertelsmann AG, die in 23 Ländern vertreten ist. Die Hälfte der Unternehmen gibt Beteiligungen in bis zu acht europäischen Ländern an. Kein einziges Unternehmen ist ausschließlich im außereuropäischen Ausland vertreten. Die Zahl der rein europäischen Unternehmen beträgt 28. Damit zeichnet sich in der regionalen Streuung eine wichtige Rolle europäischer Beteiligungen im Vergleich zum außereuropäischen Ausland ab.

Tabelle 4 listet die Unternehmen auf, die beim Index der realwirtschaftlichen Internationalisierungsdimension die höchsten Werte erzielen. Sechs unter den ersten zehn Firmen sind in der Chemieindustrie tätig. Dieses Resultat bestätigt Aussagen früherer Studien, die den chemischen Sektor in Deutschland als am stärksten internationalisiert identifizierten (Lane 1998). Wirft man einen vergleichenden Blick auf Unternehmensdaten des Jahres 1986,5 so stellt sich heraus, dass der Internationalisierungspfad von der chemischen Industrie schon früh beschritten wurde.

\section{Kapitalmarktbezogene Dimension}

35 Prozent der Unternehmen befinden sich in ausschließlich deutscher Eigentümerschaft. Die restlichen Unternehmen weisen eine mehr oder minder starke Internationalisierung ihrer Eigentümerstrukturen auf. Der durchschnittliche Anteil der Unternehmensanteile, die sich in ausländischer Hand befinden, liegt bei 16 Prozent. Unternehmen mit einem hohen Anteil an Streubesitz weisen eine besonders weit reichende Internationalisierung ihrer Aktionärsstruktur auf. An der Spitze stehen die Mannesmann AG und die Metallgesellschaft AG, für die ein ausländischer Anteil von 60 bis 70 Prozent ermittelt wurde.

30 Prozent der Unternehmen sind an ausländischen Börsen notiert. Die Spitzengruppe wird von der Bayer AG (13 Börsen), der Volkswagen AG (11), der Hoechst AG (10) sowie der Deutschen Bank AG (9) gebildet. Als besonderes Signal an die internationalen Kapitalmärkte müssen Notierungen an der New York Stock Exchange (NYSE) interpretiert werden. Unternehmen, die hier notiert sind, müssen sich der strengen US-Börsenaufsicht unterwerfen. Fünf Unternehmen aus der betrachteten Grundgesamtheit haben diesen Börsengang absolviert: die Daimler-Benz AG (schon vor der Fusion zur Daimler-Chrysler AG), die Deutsche Telekom AG, die Hoechst AG, die SAP AG und die VEBA AG.

38 Prozent der Unternehmen bilanzieren nach internationalen Vorschriften. Darunter befinden sich die meisten der DAX-30-Unternehmen. Elf der Unternehmen bilanzieren nach den besonders strengen amerikanischen General Accepted Accounting Principles (GAAP), die anderen richten sich nach den International Accounting Standards (IAS).

Tabelle 5 listet die Spitzenreiter des kapitalmarktbezogenen Internationalisierungsindex auf. Es fällt auf, dass vier der zehn Spitzenunternehmen an den großen grenz-

5 In dem Forschungsprojekt werden diese auch erhoben, sie werden jedoch an dieser Stelle nicht weiter ausgewertet. 
Abbildung 1: Internationalisierung nach realwirtschaftlicher und kapitalmarktbezogener Dimension 1996

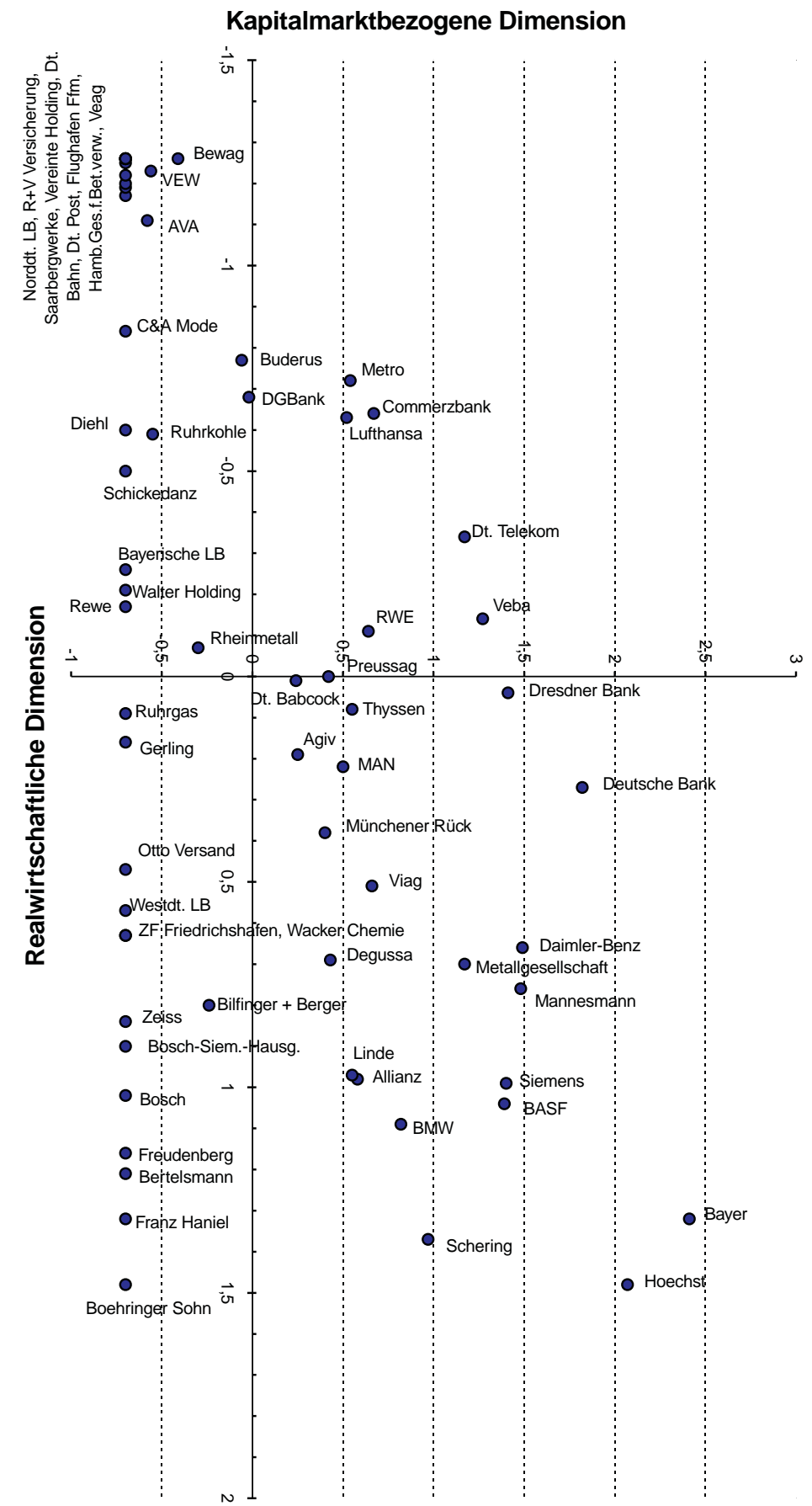


überschreitenden Fusionen beteiligt waren (Daimler-Benz AG/Chrysler, Hoechst AG/ Rhône-Poulenc, Mannesmann AG/Vodafone, Deutsche Bank AG/Bankers Trust). Zudem bereiten die VEBA AG und die VIAG AG, die ebenfalls vordere Plätze einnehmen, eine der spektakulärsten nationalen Großfusionen vor. Auch in Bezug auf Übernahmen sind kapitalmarkt-internationalisierte Unternehmen überdurchschnittlich aktiv. Diese Internationalisierungsform wirkt sich offensichtlich positiv auf die Bereitschaft zur Teilnahme an Fusionen und Übernahmen aus.

Abbildung 1 demonstriert das Zusammenspiel zwischen den beiden Dimensionen. Unternehmen wie die Bayer AG sowie die Hoechst AG erweisen sich in beide Richtungen als besonders stark internationalisiert. Andere Firmen verfolgen nur einen der beiden Pfade. Eine Reihe von Unternehmen ist realwirtschaftlich in großem Ausmaß international tätig, verbleibt aber im Familienbesitz und damit in nationaler Eigentümerschaft (z.B. Freudenberg KG). Andere wiederum streben vorrangig die Präsenz auf internationalen Kapitalmärkten an. Beispiele dafür sind die Telekom AG sowie die VEBA AG und die RWE AG, die alle ehemals staatlichem Besitz entstammen. Im linken unteren Quadranten positionieren sich die Unternehmen, die in beiden Dimensionen nicht oder nur schwach internationalisiert sind. Hier befinden sich vor allem Dienstleistungsunternehmen, die auf den Binnenmarkt ausgerichtet sind: Handelsunternehmen, regionale Energieversorger sowie die Deutsche Bahn AG und die Deutsche Post AG. Interessant ist hier die Beobachtung, wie stark sich Privatisierungsprozesse ehemals staatlicher Unternehmen unterscheiden können. Das Schlusslicht aller Unternehmen in beiden Dimensionen stellt die Norddeutsche Landesbank Girozentrale dar.

\section{Zusammenfassung: Zwei Dimensionen der Internationalisierung}

In der internationalen Diskussion zur Messung der Internationalisierungsgrade von Unternehmen wurden mehrere Vorschläge zur Konstruktion zusammengesetzter, mehrdimensionaler Internationalisierungsindizes unterbreitet. Solche Indizes verdichten Informationen und erleichtern den empirischen Unternehmensvergleich. Ein Abgleich der drei relevanten Internationalisierungsindizes zeigt aber auch die Schwierigkeiten, die mit der Konstruktion solcher Kennziffern verbunden sind. Es wurde argumentiert, dass die Verdichtung mehrerer Internationalisierungsindikatoren zu Indizes nur sinnvoll ist, wo die Indikatoren sowohl theoretisch als auch empirisch zueinander passen.

Es wurde vorgeschlagen, zwei Internationalisierungsdimensionen zu unterscheiden, die jeweils durch einen zusammengesetzten Index repräsentiert werden. Die eine Dimension besteht in der realwirtschaftlichen Internationalisierung von Produktion und Absatz über mehrere Länder hinweg. Die andere Dimension bezieht sich auf die Internationalisierung der Eigentümerstrukturen und die Übernahme anglo-amerikanischer Corporate-Governance Standards. Insbesondere der letzte Punkt verdeutlicht die Sampleabhängigkeit eines Messverfahrens. Die hier erhobene Annäherung an anglo-amerikanische Praktiken kann als Internationalisierungsindikator nur auf kontinentaleuropäische Unternehmen angewandt werden.

Den beiden Internationalisierungsformen liegen jeweils eigene Motive und Konsequenzen der unternehmerischen Strategiebildung zu Grunde. Anhand der Gruppe der 
100 größten deutschen Unternehmen wurden Indikatoren zur Messung dieser Internationalisierungsdimensionen erhoben. Die realwirtschaftliche Seite der Internationalisierung wird anhand der ausländischen Anteile an Absatz und Produktion sowie anhand der regionalen Streuung der Auslandsaktivitäten gemessen. Zur Messung der kapitalmarktbezogenen Seite der Internationalisierung wurden die Notierung an ausländischen Börsen, die Internationalität der Rechnungslegung sowie der Anteil ausländischer Aktionäre erhoben.

Es zeigen sich hohe Korrelationen zwischen den Indikatoren der jeweiligen Dimensionen, was die Indexbildung empirisch rechtfertigt. Über die Dimensionen hinweg sind die Indikatoren hingegen nur schwach korreliert. Die Idee eines universellen Internationalisierungsindex muss deshalb aufgegeben werden. Es gibt - mindestens zwei Dimensionen der Internationalisierung. Die vorgeschlagenen Internationalisierungsindikatoren bieten Anschlussmöglichkeiten in der empirischen betriebs- und sozialwissenschaftlichen Internationalisierungsforschung. Die empirischen Befunde sind auch für die Diskussion um die theoretische Modellierung von Internationalisierungsprozessen relevant. Die kapitalbezogene Internationalisierung ist weitgehend unabhängig von der güterwirtschaftlichen Internationalisierung der Unternehmen. Damit hat sich der Raum möglicher grenzüberschreitender Aktivitäten für Unternehmen auf die Übernahme von Eigentumstiteln und internationalem Kapital für alle Unternehmen ausgeweitet, auch für solche, die realwirtschaftlich nur wenig grenzüberschreitend tätig sind. Theorien, die den Prozess der Internationalisierung als eindimensionales Stufenund Phasenmodell beschreiben, sind somit zweifelhaft geworden.

\section{Literatur}

Armingeon, Klaus, 1994: Staat und Arbeitsbeziehungen. Ein internationaler Vergleich. Opladen: Westdeutscher Verlag.

Bartlett, Christopher A., 1989: Aufbau und Management der transnationalen Unternehmung: Die neue transnationale Herausforderung. S. 425-464 in: Michael Porter (Hg.): Globaler Wettbewerb: Strategien der neuen Internationalisierung. Wiesbaden: Gabler.

Bartlett, Christopher A., und Sumantra Ghoshal, 1986: Tap Your Subsidiaries for Global Reach, Harvard Business Review 64: 87-94.

Bartlett, Christopher A., und Sumantra Ghoshal, 1987a: Managing Across Borders. New Strategic Requirements, Sloan Management Review 28: 7-18.

Bartlett, Christopher A., und Sumantra Ghoshal, 1987b: Managing Across Borders. New Organizational Responses, Sloan Management Review 29: 43-54.

Bartlett, Christopher A., und Sumantra Ghoshal, 1989: Managing Across Borders: The Transnational Solution. Boston: Harvard Business School Press.

Daniels, John D., und John Bracker, 1989: Profit Performance: Do Foreign Operations Make a Difference?, Management International Review 29: 46-56.

Döhrn, Roland, 1997: Die ,Unternehmensdatenbank Globalisierung: Konzeption und erste Ergebnisse. RWI-Papier Nr. 49. Essen: Rheinisch-Westfälisches Institut für Wirtschaftsforschung.

Dörre, Klaus, 1997: Globalisierung - eine strategische Option. Internationalisierung von Unternehmen und industrielle Beziehungen in der Bundesrepublik, Industrielle Beziehungen 4: 265290.

Dörrenbächer, Christoph, 2000: Measuring Corporate Internationalisation, WZB Discussion Paper FS I 00-101, Berlin. 
Dülfer, Eberhard, 1999: Internationales Management in unterschiedlichen Kulturbereichen. München/Wien: Oldenbourg.

Germann, Harald, Bert Rürup und Martin Setzer, 1995: Globalisierung der Wirtschaft: Begriffe, Bereich und Indikatoren. Forschungsprojekt Ökonomische Dimensionen, Bereiche und Indikatoren der Globalisierung. Arbeitspapier Nr. 1 Oktober: Technische Hochschule Darmstadt.

Glaum, Martin, 1996: Internationalisierung und Unternehmenserfolg. Wiesbaden: Gabler.

Hassel, Anke, Martin Höpner, Antje Kurdelbusch, Britta Rehder und Rainer Zugehör, 2000: Dimensionen der Internationalisierung: Ergebnisse der Unternehmensdatenbank ,Internationalisierung der 100 größten Unternehmen in Deutschland'. Working Paper 1/2000. Max-Planck-Institut für Gesellschaftsforschung: Köln.

Hirsch-Kreinsen, Hartmut, 1992: Globalisierung der Industrie: Strategien, Grenzen und Folgen. S. 99-122 in: Institut für Sozialwissenschaftliche Forschung u.a. (Hg.): Jahrbuch sozialwissenschaftliche Technikberichterstattung 1996, Schwerpunkt: Reorganisation. München: edition sigma.

Hirst, Paul, und Grahame Thompson, 1996: Globalisation in Question. The International Economy and the Possibilities of Governance. Cambridge: Polity Press.

Hofstede, Geert, 1993: Cultural Constraints in Management Theories, Academy of Management Executive 1: 81-94.

Ietto-Gillies, Grazia, 1998: Different Conceptual Frameworks for the Assessment of the Degree of Internationalization: An Empirical Analysis of the Various Indices for the Top 100 Transnational Corporations, Transnational Corporations 7: 17-39.

Johanson, Jan, und Jan-Erik Vablne, 1977: The Internationalization Process of the Firm - A Model of Knowledge Development and Increasing Foreign Market Commitments, Journal of International Business Studies 8: 23-32.

Jong, Henk Wouter de, 1997: The Governance Structure and Performance of Large European Corporations, Journal of Management and Governance 1: 5-27.

Kapstein, Ethan, 1996: Workers and the World Economy, Foreign Affairs (May/June): 16-37.

Keohane, Robert O., und Helen V. Milner (Hg.), 1996: Internationalization and Domestic Politics. New York/Cambridge: Cambridge University Press.

Kochan, Thomas A., Harry C. Katz und Robert McKersie, 1986: The Transformation of American Industrial Relations. New York: Basic Books.

Lane, Christel, 1998: European Companies Between Globalization and Localization: A Comparison of Internationalization of British and German MNCs, Economy and Society 27: 462-485.

Leong, Siew Meng, und Chin Tiong Tan, 1993: Managing Across Borders: An Empirical Test of the Bartlett and Ghoshal [1989] Organizational Typology, Journal of International Business Studies 24: 449-464.

Maisonrouge, Jacques, 1983: Education of a Modern International Manager, Journal of International Business Studies 13: 56-60.

Monopolkommission, 1998: Marktöffnung umfassend verwirklichen. Zwölftes Hauptgutachten der Monopolkommission. Hauptband. Baden-Baden.

OECD, 1995: Financial Markets and Corporate Governance, Financial Market Trends 62 (Dezember): $13-35$.

Pedersen, Torben, und Steen Thomsen, 1997: European Patterns of Corporate Ownership: A Twelve Country Study, Journal of International Business Studies 4: 759-778.

Perlmutter, Howard V., 1969: The Tortuous Evolution of the Multinational Corporation, Columbia Journal of World Business 4 (Januar-Februar): 9-18.

Porta, Rafael La, und Florencio Lopez-de-Selanes, 1998: Corporate Ownership Around the World. Cambridge: National Bureau of Economic Research.

Ramaswamy, Kannan, K. Galan Kroeck und William Renforth, 1996: Measuring the Degree of Internationalization of a Firm: A Comment, Journal of International Business Studies 27: 167-177.

Riahi-Belkaoui, Ahmed, 1998: The Effects of the Degree of Internationalization on Firm Performance, International Business Review 7: 315-321.

Ronen, Simcha, und Oded Shenkar, 1985: Clustering Countries on Attidudinal Dimensions: A Review and Synthesis, Academy of Management Review 10: 435-454. 
Rürup, Bert, und Martin Setzer, 1996: ,Globalisierung - Operationalisierungs- und Meßversuche. FiWi-Arbeitspapier Nr. 3, März: Technische Hochschule Darmstadt.

Scharpf, Fritz W., 1997: Globalisierung als Beschränkung der Handlungsmöglichkeiten nationalstaatlicher Politik. MPIfG Discussion Paper 97/1, Köln.

Stopford, John M., und John H. Dunning, 1983: The World Directory of the Multinational Enterprises 1982-83. Detroit Michigan: Gales Research Company.

Streeck, Wolfgang, 1998: Einleitung: Internationale Wirtschaft, nationale Demokratie? S. 11-58 in: Ders. (Hg.): Internationale Wirtschaft, nationale Demokratie. Herausforderungen für die Demokratietheorie. Frankfurt a.M./New York: Campus.

Sullivan, Daniel, 1994: Measuring the Degree of Internationalization of a Firm, Journal of International Business Studies 25: 325-342.

Sullivan, Daniel, und Alan Bauerschmidt, 1990: Incremental Internationalization: A Test of Johanson and Vahlne's Thesis, Management International Review 30: 19-30.

Thelen, Kathleen, 2000: Why German Employers Cannot Bring Themselves to Dismantle the German Model. In: Torben Iversen, Jonas Pontusson und David Soskice (Hg.): Unions, Employers and Central Banks. New York: Cambridge University Press: 138-172.

UNCTAD, 1997: World Investment Report 1997. Transnational Corporations, Market Structure and Competition Policy. New York/Genf: Vereinte Nationen.

UNCTAD, 1999: World Investment Report 1999. Foreign Direct Investment and the Challenge of Development. New York/Genf: Vereinte Nationen.

Wortmann, Michael, 2000: Zur Logik von Wachstum und Restrukturierung multinationaler Konzerne. In: C. Cörrenbächer und Dieter Plehwe (Hg.): Grenzenlose Kontrolle? Organisatorischer Wandel und politische Macht multinationaler Unternehmen. Berlin: Edition Sigma.

Wortmann, Michael, Ulrich Bochum, Christoph Dörrenbächer und Klaus Peter Kisker, 1997: Globalisierung und internationale Mobilität deutscher Industrieunternehmen. DFG-Schwerpunkt ,Regulierung und Restrukturierung der Arbeit in den Spannungsfeldern von Globalisierung und Dezentralisierung'. Kolloquium am 6./7. Februar 1998 in Erlangen.

Korrespondenzanschrift: Dr. Anke Hassel, Max-Planck-Institut für Gesellschaftsforschung, Paulstr. 3, D-50676 Köln

E-Mail: Hassel@mpi-fg-koeln.mpg.de 